\title{
A Production Inventory Model with Shortages, Fuzzy Preparation Time and Variable Production and Demand
}

\author{
Nirmal Kumar Mahapatra ${ }^{1}$, Uttam Kumar Bera ${ }^{2}$, Manoranjan Maiti ${ }^{3}$ \\ ${ }^{1}$ Department of Mathematics, Panskura Banamali College, Panskura, India \\ ${ }^{2}$ Department of Mathematics, Bengal Institute of Technology \& Management, Santiniketan, India \\ ${ }^{3}$ Department of Applied Mathematics, Vidyasagar University, Midnapore, India \\ Email: \{nirmal_hridoy, bera_uttam, mmaiti2005\}@yahoo.co.in
}

Received February 8, 2012; revised March 10, 2012; accepted March 26, 2012

\begin{abstract}
A production inventory model is formulated for a single item. Here, demand varies with the on-hand inventory level and production price. Shortages are allowed and fully backlogged. The time gap between the decision and actual commencement of production is termed as "preparation time" and is assumed to be crisp/imprecise in nature. The set-up cost depends on preparation time. The fuzzy preparation time is reduced to a crisp interval preparation time using nearest interval approximation and following the interval arithmetic, the reduced problem is converted to a multi-objective optimization problem. Mathematical analysis has been made for single objective crisp model (Model-I). Numerical illustration have been made for both crisp (Model-I) and fuzzy (Model-II) models. Model-I is solved by generalized reduced gradient technique and multi-objective model (Model-II) by Global Criteria Method. Sensitivity analyses have been made for some parameters of Model-I.
\end{abstract}

Keywords: Fuzzy Preparation Time; Interval Number; Multi-Objective; Global Criteria Method

\section{Introduction}

After the development of EOQ model by Harris [1] in 1915, a lot of researchers have extended the above model with different types of demands and replenishment. A detailed literature is available in the text book such as Hadley and Whitin [2], Tersine [3], Silver and Peterson [4], etc. In classical inventory models, demand is normally assumed to be constant. But, now-a-days, with the invasion of the multi-nationals under the WTO agreement in the developing countries like India, Bangladesh, etc., there is a strong competition amongst the sellers to capture the market i.e. to allure the customers through different tactics. Common practices in this regard is to have attractive decorative gorgeous display of the items in the show room to create psychological pressure or to motivate the customers to buy more. Furthermore, demand of an item depends on unit production cost, i.e. it varies inversely with the unit production cost. This marketing policy is very useful for fashionable goods/fruits etc. There is some literature on the inventory models with stock-dependent demand. Several authors like Mandal and Phaujdar [5], Urban [6], Bhunia and Maiti [7], and others studied the above-mentioned type of inventory models.

\footnotetext{
*This work is supported by University Grants Commission, Govt. of
} India under the research grand No. F.PSW-096/05-06 (ERO).
Existence of Lead-time (the time gap between placement of order and the actual receipt of it) is a natural phenomenon in the field of business. So far, most of the researchers have dealt with either constant or stochastic lead-time. Elementary discussion on lead-time analysis are now-a-days available in the textbooks like Naddor [8], Magson [9], Foote, Kebriaci and Kumin [10] and others. In practice, it is difficult to predict the lead-time definitely/pricelessly and sometimes, the past records are also not available to form a probability distribution for the lead-time. Hence, the only alternative available to DM is to define the lead-time parameter imprecisely by a fuzzy number. Generally, lead time is associated with EOQ model i.e. instantaneous procurement or purchase of the lot. But, in a production system,the scenario is different. Here the time gap between the decision production and the actual commencement of production matters known as preparation time for the analysis of inventory control models. This preparation time means the time to collect the raw-materials, to arrange skilled/unskilled labors, to get machine ready for production, etc., and hence influences the set-up cost of the system. For the first time, Mahapatra and Maiti [11,12] formulated and solved production inventory models for a deteriorating/breakable item with imprecise preparation time.

Though multiobjective decision making (MODM) problems have been formulated and solved in many other 
areas like air pollution, structural analysis (cf. Rao [13]), transportation (cf. Li and Lai [14]), etc., till now few papers on MODM have been published in the field of inventory control. Padmanabhan and Vrat [15] formulated an inventory problem of deteriorating items with two objectives-minimization of total average cost and wastage cost in crisp environment (It is an environment where all input data are assumed to be deterministic, precisely defined and given.) and solved by non-linear goal programming method. Roy and Maiti [16] formulated an inventory problem of deteriorating items with two objectives, namely, maximizing total average profit and minimizing total waste cost in fuzzy environment. Mahapatra, Roy and Maiti [17], Mahapatra, Das, Bhunia and Maiti [18] formulated multi-objective multi-item inventory problems under some constraints.

In this paper, a production inventory control system for a single item is considered. Here demand is dependent on unit production cost and current stock level. Shortages are allowed and backlogged fully. Preparation time in production of the new consignment is allowed and crisp/fuzzy in nature. The setup cost is dependent on preparation time. The crisp problem for minimizing average cost is solved by generalized reduced gradient method. The problem minimizing average total cost with fuzzy preparation time is converted to a multi-objective minimization problem with the help of interval arithmetic and then it is solved by global criteria method to get pareto-optimal solution. Mathematical derivation and analysis also have been made for both single and multiobjective models. Further, the sensitivity analysis is included and two numerical illustration are provided.

\section{Interval Arithmetic}

Throughout this section lower and upper case letters denote real numbers and closed intervals respectively. The set of all positive real numbers is denoted by $R^{+}$. An order pair of brackets defines an interval

$A=\left[a_{L}, a_{R}\right]=\left\{a: a_{L} \leq a \leq a_{R}, a \in R^{+}\right\}$where $a_{L}$ and $a_{R}$ are respectively left and right limits of $A$.

Definition 2.1: Let $* \in\{+,-, ., /\}$ be a binary operation on the set of positive real numbers. If $A$ and $B$ are closed intervals then $A^{*} B=\left\{a^{*} b: a \in A, b \in B\right\}$ defines a binary operation on the set of closed intervals. In the case of division, it is assumed that $0 \notin B$. The operations on intervals used in this paper may be explicitly calculated from the above definition as

$$
\frac{A}{B}=\frac{\left[a_{L}, a_{R}\right]}{\left[b_{L}, b_{R}\right]}=\left[\frac{a_{L}}{b_{R}}, \frac{a_{R}}{b_{L}}\right]
$$

where $0 \notin B, 0 \leq a_{L} \leq a_{R}$ and $0<b_{L} \leq b_{R}$

$$
\begin{aligned}
& A+B=\left[a_{L}, a_{R}\right]+\left[b_{L}, b_{R}\right]=\left[a_{L}+b_{L}, a_{R}+b_{R}\right]
\end{aligned}
$$

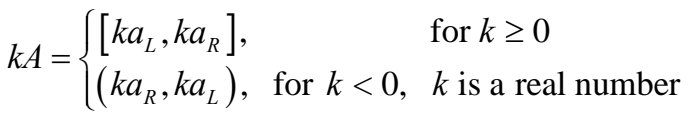

\subsection{Order Relations between Intervals}

Here, the order relations which represent the decisionmaker's preference between interval costs are defined for minimization problems. Let the uncertain costs for two alternatives be represented by intervals $\mathrm{A}$ and $\mathrm{B}$ respectively. It is assumed that the cost of each alternative is known only to lie to the corresponding interval. The order relation by the left and right limits of interval is defined in Definition-2.2.

Definition 2.2: The order relation $\leq_{L R}$ between $A=\left[a_{L}, a_{R}\right]$ and $B=\left[b_{L}, b_{R}\right]$ is defined as

$$
\begin{aligned}
& A \leq_{L R} B \text { if } a_{L} \leq b_{L} \text { and } a_{R} \leq b_{R} \\
& A<_{L R} B \text { if } A \leq_{L R} B \text { and } a_{R} \neq b_{R}
\end{aligned}
$$

The order relation $\leq_{L R}$ represents the DM's performance for the alternative with the lower minimum cost, that is, if $A \leq_{L R} B$, then $A$ is preferred to $B$.

\subsection{Formulation of the Multi-Objective Problem}

A general non-linear objective function with some interval valued parameters is as follows:

$$
\begin{aligned}
& \text { Minimize } Z(x)=\sum_{i=1}^{k} C_{i} \prod_{j=1}^{n} x_{j}^{a_{i j}} \\
& \text { subject to } \quad \sum_{i=1}^{k} A_{i} x_{j} \leq B_{j} \\
& x_{j}>0,(j=1,2, \cdots, n .), x=\left(x_{1}, x_{2}, \cdots, x_{n}\right)
\end{aligned}
$$

where $C_{i}=\left[c_{L i}, c_{R i}\right], A_{i}=\left[a_{L i}, a_{R i}\right]$ and $B_{j}=\left[b_{L j}, b_{R j}\right]$.

Now, we exhibit the formulation of the original problem (1) as a multi-objective non-linear problem. Since the objective function $Z(x)$ and the constraints contain some parameters represented by intervals, it is natural that the solution set of (1) should be defined by preference relations between intervals.

Now from Equation (1) the right and left limits $z_{R}(x), z_{L}(x)$ and centre $z_{C}(x)$ of the interval objective function $Z(x)$ respectively may be elicited as

$$
\begin{aligned}
& z_{R}(x)=\sum_{i=1}^{k} c_{L i} \prod_{j=1}^{n} x_{j}^{a_{i j}}, z_{L}(x)=\sum_{i=1}^{k} c_{L i} \prod_{j=1}^{n} x_{j}^{a_{i j}} \\
& z_{C}(x)=\frac{1}{2}\left[z_{R}(x)+z_{L}(x)\right]
\end{aligned}
$$

Thus the problem (1) is transformed into

$$
\begin{aligned}
& \text { Minimize }\left\{z_{R}, z_{C}\right\} \\
& \text { subject to } \sum_{i=1}^{k} a_{L i} x_{j} \leq b_{R j} \\
& \qquad \sum_{i=1}^{k} a_{R i} x_{j} \leq b_{L j} \\
& x_{j}>0,(j=1,2, \cdots, n), x=\left(x_{1}, x_{2}, \cdots, x_{n}\right)
\end{aligned}
$$




\subsection{Basic Fuzzy Sets and Fuzzy Numbers}

Fuzzy set: A Fuzzy set $\tilde{A}$ in a universe of discourse $X$ is defined as the following set of pairs

$\tilde{A}=\left\{\left(x, \mu_{\tilde{A}}(x)\right): x \in X\right\}$, where $\mu_{\tilde{A}}: X \rightarrow[0,1]$ is a mapping called the membership function of the fuzzy set $\tilde{A}$ and $\mu_{\tilde{A}}(x)$ is called the membership value or degree of membership of $x \in X$ in the fuzzy set $\tilde{A}$. The larger $\mu_{\tilde{A}}(x)$ is the stronger grade of membership form in $\tilde{A}$.

Fuzzy number: A fuzzy number is a fuzzy set in the universe of discourse $X$ that is both convex and normal. Figure 1 shows a fuzzy number $\tilde{A}$ of the universe of discourse $X$ that is both convex and normal.

$\alpha$-cut of a fuzzy number: The $\alpha$-cut of a fuzzy number $\tilde{A}$ is defined as crisp set

$$
A_{\alpha}=\left\{x: \mu_{\tilde{A}}(x) \geq \alpha, x \in X\right\} \text { where } \alpha \in[0,1]
$$

$A_{\alpha}$ is a non-empty bounded closed interval contained in $X$ and it can be denoted by $A_{\alpha}=\left[A_{L}(\alpha), A_{R}(\alpha)\right]$. $A_{L}(\alpha)$ and $A_{R}(\alpha)$ are the lower and upper bounds of the closed interval respectively.

Figure 1 shows a fuzzy number $\tilde{A}$ with $\alpha$-cuts $A_{\alpha_{1}}=\left[A_{L}\left(\alpha_{1}\right), A_{R}\left(\alpha_{1}\right)\right], A_{\alpha_{2}}=\left[A_{L}\left(\alpha_{2}\right), A_{R}\left(\alpha_{2}\right)\right]$. It is seen that if $\alpha_{2} \geq \alpha_{1}$ then $A_{L}\left(\alpha_{2}\right) \geq A_{L}\left(\alpha_{1}\right)$ and

$$
A_{R}\left(\alpha_{1}\right) \geq A_{R}\left(\alpha_{2}\right) \text {. }
$$

Fuzzy numbers are represented by two type of membership functions: 1) Linear membership functions e.g. Triangular fuzzy number (TFN), Trapezoidal fuzzy number, Piecewise Linear fuzzy number etc. 2) Non-linear membership functions e.g. Parabolic fuzzy number (PFN), Parabolic flat fuzzy number, Exponential fuzzy number and other non-linear fuzzy number.

\subsection{Triangular Fuzzy Number (TFN)}

TFN is the fuzzy number with the membership function $\mu_{\tilde{A}}(x)$, a continuous mapping : $\mu_{\tilde{A}}(x): R \rightarrow[0,1]$

$$
\mu_{\tilde{A}}(x)=\left\{\begin{array}{l}
0 \text { for }-\infty<x \leq a \\
1-\frac{b-x}{b-a} \text { for } a \leq x \leq b \\
1-\frac{x-b}{c-b} \text { for } b \leq x \leq c \\
0 \text { for } c \leq x<\infty
\end{array}\right.
$$

Figure 2 shows a Triangularm fuzzy number $\tilde{A}$ of the universe of discourse $X$ that is both convex and normal.

\subsection{The Nearest Interval Approximation}

Here we want to approximate a fuzzy number by crisp one. Suppose $\tilde{A}$ and $\tilde{B}$ are two fuzzy numbers with $\alpha$-cuts are $\left[A_{L}(\alpha), A_{R}(\alpha)\right]$ and $\left[B_{L}(\alpha), B_{R}(\alpha)\right]$ respectively. Then the distance between $\tilde{A}$ and $\tilde{B}$ is

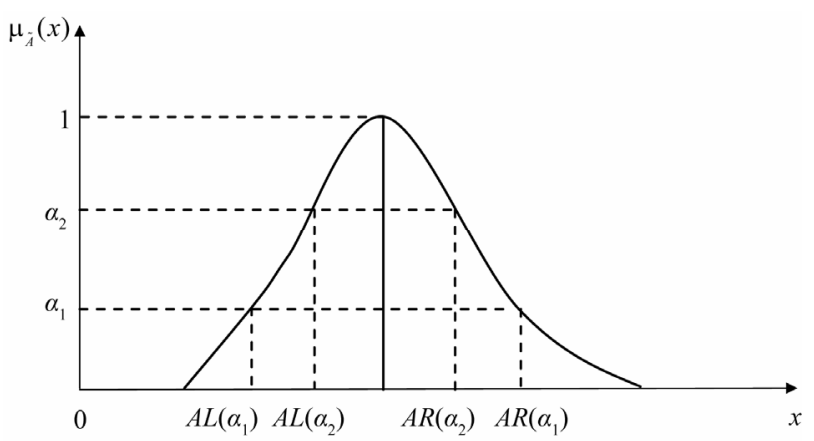

Figure 1. Fuzzy number $\tilde{A}$ with $\alpha$-cuts.

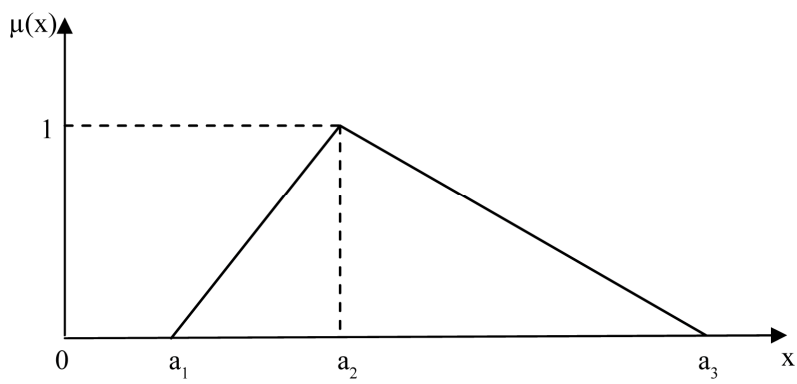

Figure 2. Triangular fuzzy number.

$$
\begin{aligned}
& \mathrm{d}(\tilde{A}, \tilde{B}) \\
& =\sqrt{\int_{0}^{1}\left(A_{L}(\alpha)-B_{L}(\alpha)\right)^{2} \mathrm{~d} \alpha+\int_{0}^{1}\left(A_{R}(\alpha)-B_{R}(\alpha)\right)^{2} \mathrm{~d} \alpha}
\end{aligned}
$$

Given $\tilde{A}$ is a fuzzy number. We have to find a closed interval $C_{d}(\tilde{A})$ which is nearest to $\tilde{A}$ with respect to metric $d$. We can do it since each interval is also a fuzzy number with constant $\alpha$-cut for all $\alpha \in[0,1]$. Hence $\left(C_{d}(\tilde{A})\right)_{\alpha}=\left[C_{L}, C_{R}\right]$. Now we have to minimize

$$
\begin{aligned}
& \mathrm{d}\left(\tilde{A}, C_{d}(\tilde{A})\right) \\
& =\sqrt{\int_{0}^{1}\left(A_{L}(\alpha)-C_{L}(\alpha)\right)^{2} \mathrm{~d} \alpha+\int_{0}^{1}\left(A_{R}(\alpha)-C_{R}(\alpha)\right)^{2} \mathrm{~d} \alpha}
\end{aligned}
$$

with respect to $C_{L}$ and $C_{R}$. In order to minimize $\mathrm{d}\left(\tilde{A}, C_{d}(\tilde{A})\right)$, it is sufficient to minimize the function $D\left(C_{L}, C_{R}\right)=\left(\mathrm{d}^{2}\left(\tilde{A}, C_{d}(\tilde{A})\right)\right)$. The first partial derivatives are

$$
\frac{\partial D\left(C_{L}, C_{R}\right)}{\partial C_{L}}=-2 \int_{0}^{1} A_{L}(\alpha) \mathrm{d} \alpha+2 C_{L}
$$

and $\frac{\partial D\left(C_{L}, C_{R}\right)}{\partial C_{R}}=-2 \int_{0}^{1} A_{R}(\alpha) \mathrm{d} \alpha+2 C_{R}$

Solving $\frac{\partial D\left(C_{L}, C_{R}\right)}{\partial C_{L}}=0$ and $\frac{\partial D\left(C_{L}, C_{R}\right)}{\partial C_{R}}=0$

we get $C_{L}^{*}=\int_{0}^{1} A_{L}(\alpha) \mathrm{d} \alpha$ and $C_{R}^{*}=\int_{0}^{1} A_{R}(\alpha) \mathrm{d} \alpha$.

Again since 


$$
\begin{aligned}
& \frac{\partial D^{2}\left(C_{L}^{*}, C_{R}^{*}\right)}{\partial C_{L}^{2}}=2>0, \frac{\partial D^{2}\left(C_{L}^{*}, C_{R}^{*}\right)}{\partial C_{R}^{2}}=2>0 \text { and } \\
& H\left(C_{L}^{*}, C_{R}^{*}\right) \\
& =\frac{\partial D^{2}\left(C_{L}^{*}, C_{R}^{*}\right)}{\partial C_{L}^{2}} \cdot \frac{\partial D^{2}\left(C_{L}^{*}, C_{R}^{*}\right)}{\partial C_{R}^{2}}-\left(\frac{\partial D^{2}\left(C_{L}^{*}, C_{R}^{*}\right)}{\partial C_{L} \cdot \partial C_{R}}\right)^{2} \\
& =4>0
\end{aligned}
$$

So $D\left(C_{L}, C_{R}\right)$ i.e. $d\left(\tilde{A}, C_{d}(\tilde{A})\right)$ is global minimum. Therefore the interval

$$
C_{d}(\tilde{A})=\left[\int_{0}^{1} A_{L}(\alpha) \mathrm{d} \alpha, \int_{0}^{1} A_{R}(\alpha) \mathrm{d} \alpha\right]
$$

is nearest interval approximation of fuzzy number $\tilde{A}$ with respect to metric $d$. Let $\tilde{A}=\left(a_{1}, a_{2}, a_{3}\right)$ be a fuzzy number. The $\alpha$-level interval of $\tilde{A}$ is defined as $(\tilde{A})_{\alpha}=\left[A_{L_{\tilde{A}}}(\alpha), A_{R}(\alpha)\right]$.

When $\tilde{A}$ is a TFN then $A_{L}(\alpha)=a_{1}+\alpha\left(a_{2}-a_{1}\right)$ and $A_{R}(\alpha)=a_{3}+\alpha\left(a_{3}-a_{2}\right)$.

By nearest interval approximation method lower and upper limits of the interval are respectively

$$
\begin{aligned}
C_{L} & =\int_{0}^{1} A_{L}(\alpha) \mathrm{d} \alpha \\
& =\int_{0}^{1}\left[a_{1}+\alpha\left(a_{2}-a_{1}\right)\right](\alpha) \mathrm{d} \alpha=\frac{1}{2}\left(a_{2}+a_{1}\right) \\
& \text { and } \\
C_{R} & =\int_{0}^{1} A_{R}(\alpha) \mathrm{d} \alpha=\int_{0}^{1}\left[a_{3}-\alpha\left(a_{3}-a_{2}\right)\right](\alpha) \mathrm{d} \alpha \\
& =\frac{1}{2}\left(a_{2}+a_{3}\right)
\end{aligned}
$$

Therefore, interval number considering $\tilde{A}$ as a TFN is $\left[\left(a_{1}+a_{2}\right) / 2,\left(a_{2}+a_{3}\right) / 2\right]$.

\section{Assumptions and Notations for the Proposed Model}

The following notations and assumptions are used in developing the model.

1) Production system involves only one non-deteriorating item.

2) Shortages are allowed and fully backlogged.

3) Time horizon is infinite.

4) $q(t)=$ inventory level at any time $t$.

5) $Q_{m}\left(Q_{s}\right)=$ the maximum inventory (shortages) level in an cycle.

6) $\tilde{L}=$ preparation time for the next production,which is a fuzzy number.

7) $C_{3}=$ Set-up cost which is of the form $C_{3}=C_{30}-C_{31} \tilde{L}^{\gamma}, 0 \leq \gamma \leq 1, \quad C_{30}$ and $C_{31}$ are two constants so chosen to best fit the set-up cost.

8) $p=$ production cost per unit item.

9) $D=$ Rate of demand which depends on production price and stock i.e.,

$$
D(p, q)=\left\{\begin{array}{l}
p^{-\varepsilon}\left(a+b q^{1-\beta}\right) \quad \text { if } q>0 \\
a p^{-\varepsilon} \quad \text { if } q \leq 0
\end{array}\right.
$$

where $a, b$ are positive real constants.

10) $t_{0}=$ cycle length of a cycle.

11) $C_{1}=$ holding cost per unit per unit time.

12) $C_{2}=$ shortage cost per unit per unit time.

13) $t^{\prime}=$ re-production time, i.e., time when production for next cycle is decided.

14) $K=$ production rate which is of the form $K=\mu D$ and $\mu \geq 1$.

\section{Mathematical Formulation}

Production inventory system involves only one item. A cycle starts with shortages at time $t=0$ and at time $t_{1}$ maximum shortages level is $Q_{s}$ and at that time production process starts to backlog the shortage quantities fully and after time $t_{2}$ the shortages reached to zero, inventory accumulates up to time $t_{3}$ of amount $Q_{m}$. At that time production process stopped, accumulated inventory declines due to demand and reaches to zero at time $t_{0}$. The above production inventory system is shown in Figure 3. The differential equations governing the stock status for this model are given by

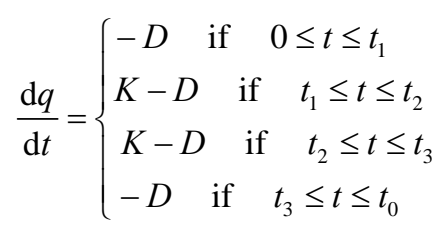

with the boundary conditions $q(0)=q\left(t_{2}\right)=q\left(t_{0}\right)=0$. These equations can be rewritten in the following form.

$$
\frac{\mathrm{d} q}{\mathrm{~d} t}=\left\{\begin{array}{l}
-a p^{-\varepsilon} \text { if } 0 \leq t \leq t_{1} \\
(\mu-1) a p^{-\varepsilon} \text { if } t_{1} \leq t \leq t_{2} \\
(\mu-1) p^{-\varepsilon}\left(a+b q^{1-\beta}\right) \quad \text { if } \quad t_{2} \leq t \leq t_{3} \\
-p^{-\varepsilon}\left(a+b q^{1-\beta}\right) \text { if } t_{3} \leq t \leq t_{0}
\end{array}\right.
$$

For simplicity we take $\beta=0$. Solving first and second equations of differential Equation (3) in the intervals $0 \leq t \leq t_{1}$ and $t_{1} \leq t \leq t_{2}$ respectively with the help of boundary conditions $q(0)=0=q\left(t_{2}\right)$, we get

$q(t)=\left\{\begin{array}{l}-a p^{-\varepsilon} t, \quad \text { from first equation of (3) } \\ -(\mu-1) a p^{-\varepsilon}\left(t_{2}-t\right), \text { from second equation of }\end{array}\right.$ with $Q_{s}=\left\{\begin{array}{l}-a p^{-\varepsilon} t_{1}, \quad \text { from first equation } \\ -(\mu-1) a p^{-\varepsilon}\left(t_{2}-t_{1}\right), \quad \text { from second equation }\end{array}\right.$

So at time $t=t_{1}$,

$$
\begin{aligned}
& -a p^{-\varepsilon} t_{1}=-(\mu-1) a p^{-\varepsilon}\left(t_{2}-t_{1}\right) \\
& \Rightarrow \mu t_{1}=(\mu-1) t_{2}
\end{aligned}
$$




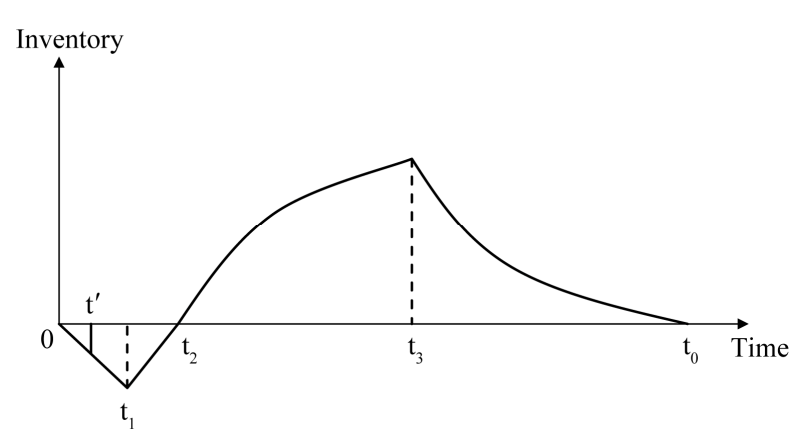

Figure 3. Inventory begins with backlogged shortages and end with no inventory.

Similarly, solving third and fourth differential Equations of (8) in the interval $t_{2} \leq t \leq t_{3}$ and $t_{3} \leq t \leq t_{0}$ respectively with the help of the boundary conditions $q\left(t_{2}\right)=0=q\left(t_{0}\right)$, we get

$q(t)=\left\{\begin{array}{l}\frac{a}{b}\left\{e^{x\left\{t-t_{2}\right\}}-1\right\}, \text { from third equation of (3) } \\ \frac{a}{b}\left\{e^{b p^{-\varepsilon}\left(t_{0}-t\right)}-1\right\}, \text { from fourth equation of (3) }\end{array}\right.$

with $Q_{m}=\left\{\begin{array}{l}\frac{a}{b}\left\{e^{x\left(t_{3}-t_{2}\right)}-1\right\} \\ \frac{a}{b}\left\{e^{b p^{-\varepsilon}\left(t_{0}-t_{3}\right)}-1\right\}\end{array}\right.$

where $x=(\mu-1) b p^{-\varepsilon}$

At time $t=t_{3}$,

$$
\begin{aligned}
& \frac{a}{b}\left\{e^{x\left(t_{3}-t_{2}\right)}-1\right\}=\frac{a}{b}\left\{e^{b p^{-\varepsilon}\left(t_{0}-t_{3}\right)}-1\right\} \\
& \Rightarrow t_{3}=\frac{t_{0}}{\mu}+t_{1} \text {, using the relation (4) }
\end{aligned}
$$

Total shortages cost $\left(S_{C}\right)$ is given by

$$
\begin{aligned}
S_{C} & =-C_{2}\left[\int_{0}^{t_{1}} q(t) \mathrm{d} t+\int_{t_{1}}^{t_{2}} q(t) \mathrm{d} t\right] \\
& =\frac{1}{2} C_{2} a p^{-\varepsilon} t_{1}^{2}+\frac{1}{2} C_{2}(\mu-1) a p^{-\varepsilon}\left(t_{2}-t_{1}\right)^{2}
\end{aligned}
$$

where $t_{1}=L+t^{\prime}, \quad t_{2}=\frac{\mu}{\mu-1} t_{1}, \quad t_{3}=\frac{t_{0}}{\mu}+t_{1}$

Now the total holding cost $\left(H_{C}\right)$ during the interval $\left[t_{2}, t_{3}\right]$ and $\left[t_{3}, t_{0}\right]$ is given by

$$
\begin{aligned}
& H_{C}=C_{1}\left[\int_{t_{2}}^{t_{3}} q(t) \mathrm{d} t+\int_{t_{3}}^{t_{0}} q(t) \mathrm{d} t\right] \\
& =\frac{C_{1} a}{b}\left[\frac{1}{x}\left\{e^{x\left(t_{3}-t_{2}\right)}-1\right\}+t_{2}\right] \\
& -\frac{C_{1} a}{b}\left[\frac{1}{b p^{-\varepsilon}}\left\{1-e^{b p^{-\varepsilon}\left(t_{0}-t_{3}\right)}\right\}+t_{0}\right]
\end{aligned}
$$

Total production cost $\left(P_{C}\right)$

$$
\begin{aligned}
& =p \int_{t_{1}}^{t_{2}} \mu a p^{-\varepsilon} \mathrm{d} t+p \int_{t_{2}}^{t_{3}} \mu p^{-\varepsilon}(a+b q) \mathrm{d} t \\
& =p^{1-\varepsilon} \mu a\left(t_{2}-t_{1}\right)+\frac{1}{x} p^{1-\varepsilon} \mu a\left\{e^{x\left(t_{3}-t_{2}\right)}-1\right\}
\end{aligned}
$$

Now the average total cost (ATC)

$$
\begin{aligned}
= & \frac{1}{t_{0}}(\text { Holding Cost }+ \text { Set up Cost } \\
& \quad+\text { Shortages Cost }+ \text { Production Cost }) \\
= & \frac{T C}{t_{0}},
\end{aligned}
$$

where

$$
\begin{aligned}
T C= & {\left[\frac{C_{1} a}{b x}\left\{e^{x\left(t_{3}-t_{2}\right)}-1\right\}+\frac{C_{1} a}{b} t_{2}\right.} \\
& -\frac{C_{1} a}{b^{2} p^{-\varepsilon}}\left\{1-e^{b p^{-\varepsilon}\left(t_{0}-t_{3}\right)}\right\} \\
& -\frac{t_{0} C_{1} a}{b}+C_{30}-C_{31} L^{\gamma} \\
& +\frac{1}{2} C_{2} a p^{-\varepsilon} t_{1}^{2}+\frac{1}{2} C_{2}(\mu-1) a p^{-\varepsilon}\left(t_{2}-t_{1}\right) \\
& \left.+p^{1-\varepsilon} \mu a\left(t_{2}-t_{1}\right)+\frac{1}{x} p^{1-\varepsilon} \mu a\left\{e^{x\left(t_{3}-t_{2}\right)}-1\right\}\right]
\end{aligned}
$$

\subsection{Crisp Model (Model-I)}

Hence, the proposed model can be stated as

Minimize $\operatorname{ATC}\left(t^{\prime}, t_{0}\right)$

$$
\begin{aligned}
= & \frac{1}{t_{0}}\left[\frac{C_{1} a}{b x}\left\{e^{x\left(t_{3}-t_{2}\right)}-1\right\}+\frac{C_{1} a}{b} t_{2}\right. \\
& -\frac{C_{1} a}{b^{2} p^{-\varepsilon}}\left\{1-e^{b p^{-\varepsilon}\left(t_{0}-t_{3}\right)}\right\} \\
& -\frac{t_{0} C_{1} a}{b}+C_{30}-C_{31} L^{\gamma} \\
& +\frac{1}{2} C_{2} a p^{-\varepsilon} t_{1}^{2}+\frac{1}{2} C_{2}(\mu-1) a p^{-\varepsilon}\left(t_{2}-t_{1}\right)^{2} \\
& \left.+p^{1-\varepsilon} \mu a\left(t_{2}-t_{1}\right)+\frac{1}{x} p^{1-\varepsilon} \mu a\left\{e^{x\left(t_{3}-t_{2}\right)}-1\right\}\right]
\end{aligned}
$$

\subsection{Fuzzy Model (Model-II)}

As in this case, preparation time is a fuzzy number $\tilde{L}$ which is replaced by an appropriate interval number $L=\left[L_{L}, L_{R}\right]$ and so, in this case $t_{1}$ is replaced by $\left[L_{L}, L_{R}\right]+t^{\prime}$ (for details see Appendix I) and the crisp problem (7) becomes a fuzzy optimization problem which, using interval arithmetic, becomes a multi-objective nonlinear programming problem as follows: 


$$
\text { Minimize } A T C_{C}, A T C_{R}
$$

where $A T C_{L}=\frac{1}{t_{0}}\left[H_{C L}+C_{3 L}+S_{C L}+P_{C L}\right]$

$$
A T C_{R}=\frac{1}{t_{0}}\left[H_{C R}+C_{3 R}+S_{C R}+P_{C R}\right]
$$

and $A T C_{C}=\frac{1}{2}\left(A T C_{L}+A T C_{R}\right)$.

\section{Mathematical Analysis}

\subsection{Model-I (Crisp Model)}

The objective function ATC of Model-I is a function of $t^{\prime}$ and $t_{0}$ as $t_{1}, t_{2}$ and $t_{3}$ are dependent variable (depend on $t^{\prime}$ and $t_{0}$ ). To achieve optimal $t^{\prime}$ and $t_{0}$, the partial derivatives of the average total cost $A T C$ with respect to $t^{\prime}$ and $t_{0}$ are set to zero. The resulting equation can be solved simultaneously to obtain the optimal $t^{\prime}$ and $t_{0}$.

$$
\begin{aligned}
& \frac{\partial A T C}{\partial t^{\prime}}=\frac{1}{t_{0}} \frac{\partial T C}{\partial t^{\prime}} \\
& =\frac{1}{t_{0}}\left[-\frac{C_{1} a}{b(\mu-1)} e^{x\left[\frac{t_{0}}{\mu}-\frac{L}{\mu-1}-\frac{t^{\prime}}{\mu-1}\right]}\right. \\
& +\frac{C_{1} a \mu}{b(\mu-1)}-\frac{C_{1} a}{b} e^{b p^{-\varepsilon\left\{\frac{\mu-1}{\mu} t_{0}-\left(L+t^{\prime}\right)\right\}}} \\
& +\frac{p^{1-\varepsilon} \mu a}{\mu-1}-\frac{p^{1-\varepsilon} \mu a}{\mu-1} e^{x\left[\frac{t_{0}}{\mu}-\frac{L}{\mu-1}-\frac{t^{\prime}}{\mu-1}\right]}+C_{2} a p^{-\varepsilon}\left(L+t^{\prime}\right) \\
& \left.+C_{2} a p^{-\varepsilon}\left(\frac{1}{\mu-1} t^{\prime}+\frac{1}{\mu-1} L\right)\right]=0
\end{aligned}
$$

and

$$
\begin{aligned}
& \frac{\partial A T C}{\partial t_{0}}=\frac{1}{t_{0}} \frac{\partial T C}{\partial t_{0}}-\frac{1}{t_{0}^{2}} T C \\
& =\frac{1}{t_{0}}\left[\frac{C_{1} a}{b \mu} e^{x\left[\frac{t_{0}}{\mu}-\frac{L}{\mu-1}-\frac{t^{\prime}}{\mu-1}\right]}\right. \\
& +\frac{C_{1} a(\mu-1)}{b \mu} e^{b p^{-\varepsilon}\left\{\frac{\mu-1}{\mu} t_{0}-\left(L+t^{\prime}\right)\right\}}-\frac{C_{1} a}{b} \\
& \left.+\frac{p^{1-\varepsilon} \mu a}{\mu} e^{x\left[\frac{t_{0}}{\mu}-\frac{L}{\mu-1}-\frac{t^{\prime}}{\mu-1}\right]}\right]-\frac{1}{t_{0}^{2}} T C=0 \\
& \text { i.e. } \frac{\partial T C}{\partial t_{0}}=\frac{1}{t_{0}} T C
\end{aligned}
$$

$A T C$ can be proved as strictly convex since all the principal minors of its Hessian matrix are strictly positive.

$$
\frac{\partial^{2} A T C}{\partial t^{\prime 2}}=\frac{1}{t_{0}} \frac{\partial T C^{2}}{\partial t^{\prime 2}},
$$

where

$$
\begin{aligned}
& \frac{\partial T C^{2}}{\partial t^{\prime 2}}=\left[\frac{C_{1} a x}{b(\mu-1)^{2}} e^{x\left[\frac{t_{0}}{\mu}-\frac{L}{\mu-1}-\frac{t^{\prime}}{\mu-1}\right]}\right. \\
& +C_{1} a p^{-\varepsilon} e^{b p^{-\varepsilon}\left\{\frac{\mu-1}{\mu} t_{0}-\left(L+t^{\prime}\right)\right\}} \\
& \left.+\frac{p^{1-\varepsilon} \mu a x}{(\mu-1)^{2}} e^{x\left[\frac{t_{0}}{\mu}-\frac{L}{\mu-1}-\frac{t^{\prime}}{\mu-1}\right]}+C_{2} a p^{-\varepsilon}+\frac{C_{2} a p^{-\varepsilon}}{\mu-1}\right]>0 \\
& \frac{\partial^{2} A T C}{\partial t_{0}^{2}}=\frac{1}{t_{0}} \frac{\partial T C^{2}}{\partial t_{0}^{2}}-\frac{2}{t_{0}^{2}} \frac{\partial T C}{\partial t_{0}}+\frac{2}{t_{0}^{3}} T C \\
& =\frac{1}{t_{0}} \frac{\partial T C^{2}}{\partial t_{0}^{2}}
\end{aligned}
$$

using (10) , where

$$
\begin{aligned}
\frac{\partial T C^{2}}{\partial t_{0}^{2}}=[ & {\left[\frac{C_{1} a x}{b \mu^{2}} e^{x\left[\frac{t_{0}}{\mu}-\frac{L}{\mu-1}-\frac{t^{\prime}}{\mu-1}\right]}\right.} \\
& +\frac{C_{1} a(\mu-1)^{2} p^{-\varepsilon}}{b \mu^{2}} e^{b p^{-\varepsilon}\left\{\frac{\mu-1}{\mu} t_{0}-\left(L+t^{\prime}\right)\right\}} \\
& \left.+\frac{p^{1-\varepsilon} \mu a x}{\mu^{2}} e^{x\left[\frac{t_{0}}{\mu}-\frac{L}{\mu-1}-\frac{t^{\prime}}{\mu-1}\right]}\right]>0
\end{aligned}
$$

and

$$
\begin{aligned}
& \frac{\partial^{2} A T C}{\partial t^{\prime 2}} \times \frac{\partial^{2} A T C}{\partial t_{0}^{2}}-\left\{\frac{\partial^{2} A T C}{\partial t_{0} \partial t^{\prime}}\right\}^{2} \\
& =\frac{1}{t_{0}^{2}}\left\{\frac{\partial^{2} T C}{\partial t^{\prime 2}} \times \frac{\partial^{2} T C}{\partial t_{0}^{2}}-\left\{\frac{\partial^{2} T C}{\partial t_{0} \partial t^{\prime}}\right\}^{2}\right\}
\end{aligned}
$$

using (11) and (12) $=\left\{C_{2} a p^{-\varepsilon}+\frac{C_{2} a p^{-\varepsilon}}{\mu-1}\right\} \times \frac{\partial^{2} A T C}{\partial t_{0}^{2}}>0$

Hence, the solution of Model-I is global minimum of $A T C$.

\subsection{Model-II (Fuzzy Model)}

In Model-II, the objective functions $A T C_{L}$ and $A T C_{R}$ (and hence $A T C_{C}$ ) are functions of $t^{\prime}$ and $t_{0}$ as $t_{1}, t_{2}$ and $t_{3}$ are dependent variable (depend on $t^{\prime}$ and $t_{0}$ ). As of Model-I, it can be seen that $A T C_{L}$ and $A T C_{R}$ (and hence $A T C_{C}$ ) are strictly convex.

Definition 5.1: A multi-objective optimization problem is convex if all the objective functions and the feasible region are convex.

Definition 5.2: $\left[t^{\prime *}, t_{0}^{*}\right]$ is said to be a Pareto optimal solution to the MONLP iff there does not exist another $\left[t^{\prime}, t_{0}\right]$ in the feasible region such that $A T C_{i}\left(t^{\prime}, t_{0}\right) \leq A T C_{i}\left(t^{\prime *}, t_{0}^{*}\right)$ for all $i(i=C, R)$ and 
$A T C_{j}\left(t^{\prime}, t_{0}\right)<A T C_{j}\left(t^{\prime *}, t_{0}^{*}\right)$ for at least one index $j(j=C, R)$.

An objective vector $\left(A T C_{C}^{*}, A T C_{R}^{*}\right)$ is Pareto-optimal if there does not exist another objective vector $\left(A T C_{C}, A T C_{R}\right)$ such that $A T C_{i} \leq A T C_{i}^{*}$ for all $i=C, R$ and $A T C_{j}<A T C_{j}^{*}$ for at least one index $j=C, R$. Therefore, $\left(A T C_{C}^{*}, A T C_{R}^{*}\right)$ is Pareto-optimal if the decision vector corresponding to it is Pareto optimal.

Theorem 5.1: Let the multi-objective optimization problem be convex. Then every locally Pareto-optimal solution is also globally Pareto-optimum (cf. Miettinen [19])).

Therefore the problem given by (7) is a strictly convex multi-objective minimization problem and hence possesses global Pareto-optimal solutions. Hence, the problem (7) is solved by Global Criteria Method.

\subsection{Global Criteria Method}

The Multi-Objective Non-linear Programming (MONLP) problems are solved by Global Criteria Method converting it to a single objective optimization problem. The solution procedure is as follows.

- Step-1. Solve the multi-objective programming problem (16) as a single objective problem using only one objective at a time ignoring.

- Step-2. From the above result, formulate a pay-off matrix as follows:

$$
\left(\begin{array}{ll}
A T C_{C}^{\min } & A T C_{R}^{\max } \\
A T C_{C}^{\max } & A T C_{R}^{\min }
\end{array}\right)
$$

- Step-3. From the pay-off matrix of Step-2, determine the ideal objective vector, say $\left(A T C_{C}^{\min }, A T C_{R}^{\min }\right)$ and the corresponding value of $\left(A T C_{C}^{\max }, A T C_{R}^{\max }\right)$. Here, the ideal objective vector is used as a reference point. The problem is then to solve the following auxiliary problem:

$$
\begin{aligned}
G C & =\text { Minimize }\left\{\left(\frac{A T C_{C}\left(t^{\prime}, t_{0}\right)-A T C_{C}^{\min }}{A T C_{C}{ }^{\text {max }}-A T C_{C}^{\min }}\right)^{p}\right. \\
& \left.+\left(\frac{A T C_{R}\left(t^{\prime}, t_{0}\right)-A T C_{R}{ }^{\min }}{A T C_{R}{ }^{\text {max }}-A T C_{R}{ }^{\text {min }}}\right)^{p}\right\}^{\frac{1}{p}}
\end{aligned}
$$

where $1 \leq p<\infty$ and $G C$ is known as global criteria. An usual value of $p$ is 2 .

\section{Numerical Illustrations}

\subsection{Crisp Model (Model-I)}

The Equation (7) is non-linear in $t^{\prime}$ and $t_{0}$ and difficult to obtain the optimal values of $t^{\prime}$ and $t_{0}$ by
Calculus method. Hence, we minimize (7) by a gradient based non-linear optimization method. Using the optimal values of $t^{\prime}$ and $t_{0}$, the corresponding values of $t_{1}, t_{2}, t_{3}, Q_{s}, Q_{m}$ and minimum average total cost (ATC) are be obtained. To illustrate the proposed inventory model (Model-I), following input data are considered.

$$
\begin{aligned}
& L=0.6, \mu=1.8, a=300, b=2, \varepsilon=0.7, \\
& \gamma=0.5, C_{1}=1.5, p=25, C_{30}=2000, \\
& C_{31}=300, C_{2}=15 \text { in appropriate units. }
\end{aligned}
$$

Solving the Model-I, following optimal values are obtained:

$$
\begin{aligned}
& A T C=1356.35, t^{\prime}=0.6001609, t_{0}=6.939239, \\
& Q_{s}=37.82707, Q_{m}=72.84892
\end{aligned}
$$

\subsection{Fuzzy Model (Model-II)}

To illustrate the proposed inventory Model-II, following input data are considered.

$$
\begin{aligned}
& L_{L}=0.5, L_{R}=0.8, \mu=1.8, a=300, b=2, \varepsilon=0.7, \\
& \gamma=0.5, C_{1}=1.5, p=25, C_{30}=2000, C_{31}=300, \\
& C_{2}=15 \text { in appropriate units. }
\end{aligned}
$$

For the above input data, formulate a pay-off matrix as follows:

$$
\begin{aligned}
& A T C_{C} \quad A T C_{R} \\
& \left(\begin{array}{ll}
1364.005 & 1753.147 \\
1385.503 & 1722.588
\end{array}\right)
\end{aligned}
$$

Solving the Model-II using Global Criteria Method, following optimal values are obtained:

$$
\begin{aligned}
& A T C_{C}=1369.40, A T C_{R}=1730.23, A T C_{L}=1008.61, \\
& t^{\prime}=0.6327567, t_{0}=7.941731, G C=0.3543743
\end{aligned} .
$$

\section{Discussion}

The Model-I is a single objective optimization problem and is solved using generalized reduced gradient method. The Model-II is a multiobjective problem and is solved by Global Criteria Method. In Model-II, the average cost minimization problem with interval objective function was converted into the multi-objective problem whose objectives are to minimize the centre $\left(A T C_{C}\right)$ and right limit $\left(A T C_{R}\right)$ of the interval objective function. These two objective functions can be considered as the minimization of the average case and the worst case. The solution sets of production inventory problems with interval objective functions are defined as the Pareto optimal solutions of the corresponding multi objective problem. Therefore, the solution set defined in this paper includes the optimal solutions against both the worst and the average cases. 
Table 1. Sensitivity analysis of Model-I.

\begin{tabular}{|c|c|c|c|c|c|c|}
\hline parameter & $\%$ changes & $\%$ of $A T C^{*}$ & $t^{\prime *}$ & $t_{0}^{*}$ & $Q_{s}$ & $Q_{m}$ \\
\hline \multirow{4}{*}{$a$} & -50 & -42.09005 & 1.051922 & 9.5283 & 26.03292 & 54.05133 \\
\hline & -20 & -16.43247 & 0.7275575 & 7.693093 & 33.47436 & 66.22929 \\
\hline & +20 & +16.07881 & 0.506768 & 6.373380 & 41.86019 & 78.68090 \\
\hline & +50 & +39.71182 & 0.4040906 & 5.737264 & 47.47090 & 86.34708 \\
\hline \multirow{4}{*}{$\mu$} & -50 & No solution & $\ldots$ & $\ldots$ & $\ldots$ & $\ldots$ \\
\hline & -20 & -4.761578 & 0.4635499 & 8.362338 & 33.52132 & 55.21444 \\
\hline & +20 & +2.550066 & 0.6733307 & 6.306766 & 40.13326 & 83.86810 \\
\hline & +50 & +4.740787 & 0.7361900 & 5.817491 & 42.11448 & 94.57485 \\
\hline \multirow{4}{*}{$L$} & -50 & +0.7166992 & 0.9207250 & 7.061990 & 38.47522 & 74.44632 \\
\hline & -20 & +0.2596661 & 0.7276148 & 6.983796 & 38.06201 & 73.42793 \\
\hline & +20 & -0.2364422 & 0.4733806 & 6.898645 & 37.61337 & 72.32223 \\
\hline & +50 & -0.5588499 & 0.2841310 & 6.843172 & 37.32184 & 71.60373 \\
\hline \multirow{4}{*}{$p$} & -50 & -2.621729 & 0.2851214 & 5.590982 & 45.31972 & 108.9680 \\
\hline & -20 & -1.285355 & 0.4873798 & 6.480728 & 40.06651 & 83.00596 \\
\hline & +20 & +1.360556 & 0.7014259 & 7.332111 & 36.10404 & 65.42835 \\
\hline & +50 & +3.408616 & 0.8376034 & 7.836238 & 34.11446 & 57.30315 \\
\hline
\end{tabular}

\section{Sensitivity Analysis}

A sensitivity analysis of the decision variables $t^{\prime}$ and $t_{0}$ for Model-I have been made when each of the parameters $a, \mu, L, p$ being changed from $-50 \%$ to $+50 \%$. The relative change of $A T C^{*}, Q_{s}^{*}, Q_{m}^{*}$ are also taken into account and presented in Table 1. From Table 1, it is seen that $A T C$ changes rapidly with the rapid change of the parameter $a$, but these change are too slow in case of parameter $\mu$ and $p$. Again, ATC increases and decreases with decrease and increase in preparation time $L$ respectively, but this change is slow which agrees with the real phenomenon.

Figure 4 shows the $A T C$ versus $b$ graph. From this graph it is revealed that initially $A T C$ decreases rapidly with the increase in $b$ and after some time it begins to increase slowly. Here, it is interesting to note the effect of shape parameter $b$ of the demand function on $A T C$.

\section{Practical Implications}

In this preparation time has been considered for an inventory model with finite production rate. In real life, once production is discontinued, i.e., once the labour force is dismantled, supply of raw materials is disrupted, machineries is kept in disorder, etc., it is obvious that to start the next production, some time is required to bring the above mentioned things in order. The cost related to above factors depends on the time gap between the decision to start the preparation and actual commence of production. If the gap is small, then everything will have to be arranged hurriedly and it costs more. Hence, setup

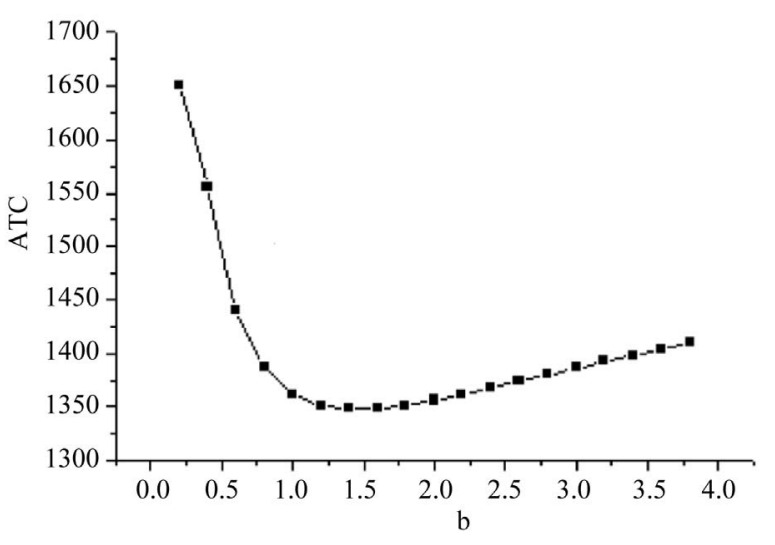

Figure 4. Graph of $A T C$ versus $b$.

cost decreases with the increase of preparation time. The preparation time for the next production run is very important. This decision influences several costs like setup cost, production cost, etc. Such real life models are considered here taking different environments into consideration.

The fuzzy numbers are described by linear/non-linear type membership functions. Fuzzy number describing preparation time is then approximated to an interval number. Following this, the fuzzy problem is converted into multi-objective inventory problem where the objective functions are represented by right limit and centre of interval function which are to be minimized. The proposed model has a broad area of applicability. Two inventory models are presented with different type of preparation time. If a decision maker (DM) knows definitely 
the time required to restart the next production run, he/ she may adopt Model-I. Similarly, for fuzzy preparation time, DM may accept the Model-II. DM may select any of these models according to the prevailing environment in his/her production centre.

\section{Conclusions}

The present paper proposes a solution procedure for production inventory model with production cost and on hand inventory dependent demand rate and preparation time. Here, shortages are allowed and backlogged fully. Like lead time, time when the decision is taken for the preparation of next production run i.e. preparation time has been considered for a production inventory model. In real life, setup cost decreases with the increase of preparation time. This consideration is taken into account in Model-I. In Model-II, preparation time is taken as imprecise via a fuzzy number. The fuzzy number is described by linear/non-linear type membership function.

Fuzzy number describing preparation time is then approximated to an interval number. Following this, the problem is converted into multi-objective inventory problem where the objective functions are represented by centre and right limit of interval function which are to be minimized. To obtain the solution of the multi-objective inventory problem, Global Criteria Method has been used. The proposed demand has a broad area of applicability. Demand of a commodity decreases with the increase in production cost but increases with the increase of stock of the displayed commodity and vice versa. Here, though the formulation of the model and the solution procedure are quite general, the model is a simple production model with demand dependent production rate. The unit production cost which is assumed here to be constant, in reality, varies with the preparation time and produced quantity. Moreover, time dependent production rate, partially lost sales, inflation, etc., can be incorporated to the model to make it more realistic. Here, demand is stockdependent. The present analysis can be repeated for the dynamic demand also. Though the problem has been presented in crisp and fuzzy environment, it can also be formulated and solved in fuzzy-stochastic environment representing production cost and inventory costs through probability distribution.

\section{REFERENCES}

[1] F. Harris, "Operations and Cost (Factory Management Series),” A.W. Shaw Co., Chicago, 1915, pp. 48-52.

[2] G. Hadley and T. M. Whitin, "Analysis of Inventory Systems,” Prentice Hall, Englewood Cliffs, 1963.

[3] R. J. Tersine, "Principles of Inventory and Materials Management,” Elsevier North Holland Inc., New York, 1982.

[4] E. A. Silver and R. Peterson, "Decision System for In- ventory Management and Research,” John Wiley, New York, 1985.

[5] B. N. Mandal and S. Phaujdar, "A Note on Inventory Model with Stock-Dependent Consumption Rate," $O P$ SEARCH, Vol. 26, 1989, pp. 43-46.

[6] T. L. Urban, "Deterministic Inventory Models Incorporating Marketing Decisions," Computer and Industrial Engineering, Vol. 22, No. 1, 1992, pp. 85-93. doi:10.1016/0360-8352(92)90035-I

[7] A. K. Bhunia and M. Maiti, “An Inventory Model for Decaying Items with Selling Price, Frequency of Advertisement and Linearly Time Dependent Demand with Shortages,” IAPQR Transactions, Vol. 22, 1997, pp. 4149.

[8] E. Naddor, "Inventory System,” John Wiley, New York, 1966.

[9] D. Magson, "Stock Control When Lead-Time Can Not Be Considered Constant," Journal of the Operational Research Society, Vol. 30, 1979, pp. 317-322.

[10] B. Foote, N. Kebriaci and H. Kumin, "Heuristic Policies for Inventory Ordering Problems with Long and Random Varying Lead Times,” Journal of Operations Management, Vol. 7, No. 3-4, 1988, pp. 115-124. doi:10.1016/0272-6963(81)90008-5

[11] N. K. Mahapatra and M. Maiti, "Inventory Model for Breakable Item with Uncertain Preparation Time,” Tamsui Oxford Journal of Management Sciences, Vol. 20, No. 2, 2004, pp. 83-102.

[12] N. K. Mahapatra and M. Maiti, "Production-Inventory Model for a Deteriorating Item with Imprecise Preparation Time for Production in Finite Time Horizon," Asia Pacific Journal of Operations Research, Vol. 23, No. 2, 2006, pp. 171-192.

[13] S. S. Rao, "Multi Objective Optimization in Structural Design with Uncertain Parameters and Stochastic Process,” AIAA Journal, Vol. 22, 1984.

[14] L. Li and K. K. Lai, "A Fuzzy Approach to the Multiobjective Transportation Problem,” Computers \& Operations Research, Vol. 27, No. 1, 2000, pp. 43-57. doi:10.1016/S0305-0548(99)00007-6

[15] G. Padmanabhan and P. Vrat, "EOQ Models for Perishable Items under Stock-Dependent Selling Rate,” European Journal of Operations Research, Vol. 86, No. 2, 1995, pp. 281-292. doi:10.1016/0377-2217(94)00103-J

[16] T. K. Roy and M. Maiti, "Multi-Objective Inventory Models of Deteriorating Items with Some Constraints in a Fuzzy Environment," Computers and Operations Research, Vol. 25, No. 12, 1998, pp. 1085-1095. doi:10.1016/S0305-0548(98)00029-X

[17] N. K. Mahapatra, T. K. Roy and M. Maiti, "Multi-Objective Multi-Item Inventory Problem," Proceedings of the Seminar on Recent Trends and Developments in Applied Mathematics, Howrah, 3 March 2001, pp. 44-68.

[18] N. K. Mahapatra, K. Das, A. K. Bhunia and M. Maiti, "Multiobjective Inventory Model of Deteriorating Items With Ramp Type Demand Dependent Production, Setup and Unit Costs," Proceedings of the National Symposium on Recent Advances of Mathematics and Its Applications 
in Science and Society, University of Kalyani, Kalyani, 21-22 November 2002, pp. 89-110.
[19] K. M. Miettinen, "Non-Linear Multi-Objective Optimization,” Kluwer’s International Series, Boston, 1999.

\section{Appendix I}

Since preparation time is a fuzzy number which is replaced by an appropriate interval number $L=\left[L_{L}, L_{R}\right]$, therefore from Equations (4) and (6), replacing $t_{1}$ by $\left(\left[L_{L}, L_{R}\right]+t^{\prime}\right)$, we have

$$
\begin{aligned}
& t_{1}=\left[L_{L}, L_{R}\right]+t^{\prime}=\left[t_{1 L}, t_{1 R}\right], t_{2}=\frac{\mu}{\mu-1} t_{1}=\left[t_{2 L}, t_{2 R}\right], \\
& t_{3}=\frac{t_{0}}{\mu}+t_{1}=\left[t_{3 L}, t_{3 R}\right]
\end{aligned}
$$

where

$$
t_{1 i}=L_{i}+t^{\prime}, t_{2 i}=\frac{\mu}{\mu-1} t_{2 i}, t_{3 i}=\frac{t_{0}}{\mu}+t_{1 i},(i=L, R)
$$

Total shortages cost $\left(S_{C}\right)$ is given by

$$
S_{C}=\left[S_{C L}, S_{C R}\right]
$$

where

$$
S_{C L}=\left[\frac{1}{2} C_{2} a p^{-\varepsilon} t_{1 L}^{2}+\frac{1}{2} C_{2} y t_{2 L}^{2}-C_{2} y t_{2 R} t_{1 R}+\frac{1}{2} C_{2} y t_{1 L}^{2}\right]
$$

Now the Set-up cost $C_{3}$ is given by

$$
C_{3}=\left[C_{30}-C_{31} L_{R}^{\gamma}, C_{30}-C_{31} L_{L}^{\gamma}\right]=\left[C_{3 L}, C_{3 R}\right] .
$$

Now the total holding cost $\left(H_{C}\right)$ during the interval $\left[t_{2}, t_{3}\right]$ and $\left[t_{3}, t_{0}\right]$ is given by

$$
H_{C}=\left[H_{C L}, H_{C R}\right] \text {, say }
$$

where

$$
\begin{aligned}
H_{C L}= & \frac{C_{1} a}{b x}\left\{e^{x\left(t_{3 L}-t_{2 R}\right)}-1\right\}+\frac{C_{1} a}{b} t_{2 L} \\
& -\frac{C_{1} a}{b^{2} p^{-\varepsilon}}\left\{1-e^{b p^{-\varepsilon}\left(t_{0}-t_{3 R}\right)}\right\}-\frac{t_{0} C_{1} a}{b}
\end{aligned}
$$

Total Production cost is given by

$$
P_{C}=\left[P_{C L}, P_{C R}\right]
$$

where

$$
P_{C L}=p^{1-\varepsilon} \mu a\left(t_{2 L}-t_{1 R}\right)+\frac{p^{1-\varepsilon} \mu a}{x}\left[e^{x\left(t_{3 L}-t_{2 R}\right)}-1\right]
$$

Now the expressions of $S_{C R}, H_{C R}$ and $P_{C R}$ may be obtained from the expressions of $S_{C L}, H_{C L}$ and $P_{C L}$ on replacing the suffices $L$ by $R$ and $R$ by $L$ respectively. Therefore, the total cost becomes

$$
\begin{aligned}
& A T C \\
= & \frac{1}{t_{0}}\left[H_{C}+C_{3}+S_{C}+P_{C}\right] \\
= & \frac{1}{t_{0}}\left[\left(H_{C L}, H_{C R}\right)+\left(C_{3 L}, C_{3 R}\right)+\left(S_{C L}, S_{C R}\right)+\left(P_{C L}, P_{C R}\right)\right] \\
= & {\left[A T C_{L}, A T C_{R}\right], }
\end{aligned}
$$

where

$$
\begin{aligned}
& A T C_{L}=\frac{1}{t_{0}}\left[H_{C L}+C_{3 L}+S_{C L}+P_{C L}\right] \\
& A T C_{R}=\frac{1}{t_{0}}\left[H_{C R}+C_{3 R}+S_{C R}+P_{C R}\right]
\end{aligned}
$$

and

$$
A T C_{C}=\frac{1}{2}\left[A T C_{L}+A T C_{R}\right] .
$$

